\title{
A study on outdoor localization method based on deep learning using model- based received power estimation data of low power wireless tag
}

\author{
Takuto Jikyo $^{1 \text { a) }}$, Takahiro Yamanishi ${ }^{1}$, Tomio Kamada ${ }^{2}{ }^{2}$, \\ Ryo Nishide $\mathbb{(}^{2}$, Chikara Ohta $\mathbb{1}^{1}$, Kenji Oyama $\mathbb{\circledR}^{3}$, \\ and Takenao Ohkawa ${ }^{2}$ \\ ${ }^{1}$ Graduate School of Science, Technology and Innovation, Kobe University, \\ 1-1 Rokkodai-cho, Nada-ku, Kobe, Hyogo 657-8501, Japan \\ ${ }^{2}$ Graduate School of System Informatics, Kobe University, \\ 1-1 Rokkodai-cho, Nada-ku, Kobe, Hyogo 657-8501, Japan \\ ${ }^{3}$ Graduate School of Agricultural Science, Kobe University, \\ 1-1 Rokkodai-cho, Nada-ku, Kobe, Hyogo 657-8501, Japan \\ a)jikyo@fine.cs.kobe-u.ac.jp
}

Abstract: We are developing a method to acquire position information of a cow outdoors using Received Signal Strength Indicator (RSSI) of Bluetooth Low Energy (BLE). As existing research, there is a localization method using fingerprint database as learning data in deep learning. However, that method has the problem that it costs to create a database by measurement in a vast outdoor environment. Therefore, we considered to build a part of the fingerprint database using virtual space modeling received power measurement environment in a pasture. Experimental results showed that an average distance error to GPS data is about $6 \mathrm{~m}$ by training DNN using the database and additionally training DNN using actual GPS data.

Keywords: BLE, RSSI, localization, deep learning, virtual space Classification: Sensing

\section{References}

[1] Y. Yamauchi, R. Nishide, Y. Takaki, C. Ohta, K. Oyama, and T. Ohkawa, "Cattle community extraction using the interactions based on synchronous behavior," Proc. of SoICT, pp. 227-234, Dec. 2018. DOI:10.1145/3287921. 3287941

[2] Space Policy Committee, "About the situation of each country positioning satellite," Cabinet Office, https://www8.cao.go.jp/space/comittee/dai68/ siryou3.pdf, accessed May 30, 2019.

[3] ARKNAV, “K-18U GPS data logger Japanese manual," International Development and Engineering Associates, https://www.ida-japan.co.jp/GPS/K18U/ 
K-18U-j-20140527.pdf, accessed May 30, 2019.

[4] NTT TechnoCross Corporation, "Active RFID product mobicollet," https:// www.ntt-tx.co.jp/products/mobicollet/, accessed May 30, 2019.

[5] Q. Li, W. Li, W. Sun, J. Li, and Z. Liu, "Fingerprint and assistant nodes based Wi-Fi localization in complex indoor environment," IEEE Access, vol. 4, pp. 2993-3004, June 2016. DOI:10.1109/ACCESS.2016.2579879

[6] R. Faragher and R. Harle, "Location fingerprinting with bluetooth low energy beacons," IEEE J. Sel. Areas Commun., vol. 33, no. 11, pp. 2418-2428, Nov. 2015. DOI:10.1109/JSAC.2015.2430281

[7] K. S. Kim, S. Lee, and K. Huang, “A scalable deep neural network architecture for multi-building and multi-floor indoor localization based on Wi-Fi fingerprinting," Big Data Analytics, vol. 3, p. 4, Apr. 2018. DOI:10.1186/ s41044-018-0031-2

[8] F. S. Daniș and A. T. Cemgil, "Model-based localization and tracking using bluetooth low-energy beacons," Sensors, vol. 17, no. 11, p. 2484, Nov. 2017. DOI: $10.3390 / \mathrm{s} 17112484$

[9] W. Zhang, K. Liu, W. Zhang, Y. Zhang, and J. Gu, "Wi-Fi positioning based on deep learning," 2014 IEEE International Conference on Information and Automation (ICIA), pp. 1176-1179, July 2014. DOI:10.1109/ICInfA.2014. 6932827

[10] T. Jikyo, T. Yamanishi, Y. Takaki, R. Nishide, T. Kamada, C. Ohta, K. Oyama, and T. Ohkawa, "A study on outdoor localization method based on deep learning using model-based received power estimation data of power-saving wireless tag," Proc. IEICE General Conference 2019, B-18-6, p. 356, Mar. 2019.

[11] A. Goldsmith, Wireless Communications, Cambridge University, the United States of America, 2005.

\section{Introduction}

In a recent, analysis of interaction among grazing Japanese black cows succeed in correctly predicting the condition such as estrus compared with sensing of the individual cow [1]. Such interaction is extracted based on location information by a Global Positioning System (GPS) device attached to each cow. Its power consumption is, however, generally large in compensation for the accuracy of 5 through 10 meters [2]. For example, in [1], an 11,000 mAh battery runs out in about 220 hours in the case that ARKNAV K-18U GPS [3] logs every five seconds. This results in frequent replacement of the battery, which will burden farmers. Therefore, we considered estimating positions using received signal strength indicator (RSSI) from Bluetooth Low Energy (BLE) tags attached to each cow. As the BLE tag system, we adopted NTT TechnoCross mobicollet, which can acquire data at intervals of two seconds and is guaranteed to operate for over a year with 220 mAh Maxell CR2032 button battery [4]. In this article, we will report the results of our research works since they may be beneficial in estimating location outdoor even for other applications.

In this study, we take a fingerprint-based approach $[5,6,7,8,9]$ using a database that stores the relationship between RSSI and position as an existing study of localization using low power wireless systems. This approach can be divided into "matching approach" and "deep learning approach." The matching approach is 
a method using pattern matching between RSSI at the time of localization and RSSI in the database $[5,6]$ and the deep learning approach is a method using the database as learning data in deep learning [7, 8, 9]. However, to realize accurate estimation over a vast outdoor environment inevitably leads to the large size of the fingerprint database, so that the matching approach will suffer from a long retrieval time in the database. In addition, as explained later, owing to the directionality of antenna of BLE tag, the different fingerprint data are required for the different postures of a cow. This means that to build the fingerprint database much costs in such a case.

Therefore, we considered creating a fingerprint database using virtual space which models received power measurement environment in the pasture. We realized outdoor localization with an average distance error of about $6 \mathrm{~m}$ by training DNN using the database and additionally training DNN with real space data. Moreover, we clarified that the deep learning approach can estimate the position more accurately and faster than the matching approach. This paper is an improvement on the method of selecting real space data used for additional learning in [10].

\section{Localization method based on deep learning using virtual space}

An outline of the localization system is given in Fig. 1. The position is estimated by inputting RSSIs, which are obtained by receivers surrounding the pasture, into the learned DNN and outputting the position coordinates by forwarding propagation. As the learning method of DNN, first, we create a received power estimation database for various posture patterns of a cow in virtual space and train DNN. Next, in order to correct the feature of received power acquired in virtual space and real space, additional learning is performed using measured RSSI data.

In preparation for localization, we attached four BLE tags to the neck of each cow, two each on the left and the right. Twenty directional antennas surround the pasture that extends about $144 \mathrm{~m}$ east to west and $82 \mathrm{~m}$ north to south. DNN was designed with RSSI of the beacon signal transmitted from each of four tags received by each of the 19 receivers except one fault receiver No. 8 as the input and the corresponding position coordinate $(x, y)$ as the output.

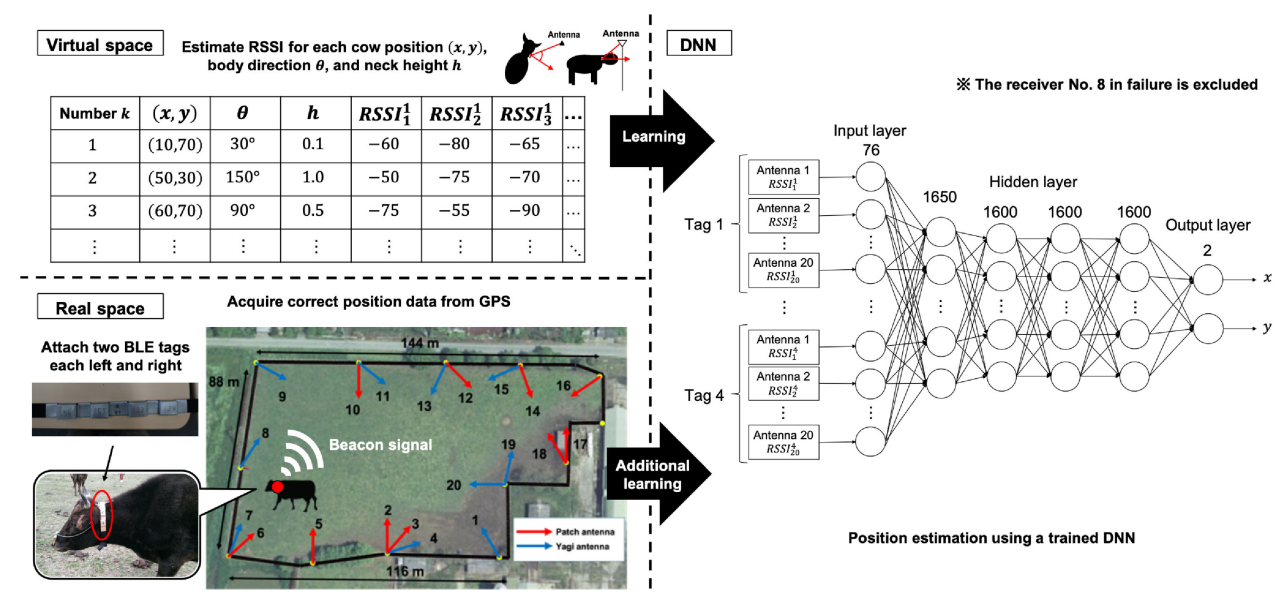

Fig. 1. Localization system. 


\subsection{Virtual space}

In what follows, we explain the radio wave propagation model, the antenna directivity of the tag, and the antenna directivity of the receiver, which were implemented in the virtual space.

Since the pasture is regarded as an open space with only a few building nearby, we applied the two-ray ground-reflection model without approximation, which is given as (2.12) of [11], and then we confirmed that it coincides the measured data well.

We also obtained the antenna directionality of the tag by measuring the RSSI at the receiver $1.5 \mathrm{~m}$ apart from the tag in an anechoic chamber. More precisely, during the measurement, we rotated the tag horizontally every $15^{\circ}$ around while it was fixed vertically. And then, we rotated the tag $10^{\circ}$ in the vertical direction. After repeating this operation, we interpolated RSSIs using cubic spline interpolation for unmeasured directions.

As for the directional modeling of the patch antenna and 8-element Yagi antenna, we calculated the gain pattern by multiplying horizontal $360^{\circ}$ and vertical $180^{\circ}$ from the gain pattern of E-plane and H-plane using the $2.5 \mathrm{D}$ antenna model of the commercial network simulator Scenargie. Besides, the gain of the patch antenna used in this research is $8 \mathrm{dBi}$, the E-plane Half Width at Half Maximum (HWHM) is $31^{\circ}$, and the H-plane HWHM is $38^{\circ}$ and the gain of the 8-element Yagi antenna is $12 \mathrm{dBi}$, the E-plane HWHM is $21^{\circ}$, and the H-plane HWHM is $24^{\circ}$.

\subsection{Virtual data}

The fingerprint database was created by estimating RSSI using the model of virtual space for each of a total of $2,769,840$ patterns of body orientation 24 patterns in $15^{\circ}$ steps, neck height 10 patterns from 0.1 to $1.0 \mathrm{~m}$ in $0.1 \mathrm{~m}$ steps, and 11,541 patterns that changed the position in $1 \mathrm{~m}$ steps from east to west in the whole range.

\subsection{Real data}

As real data, we used data of the cow with an individual number 20112 for a total of 10 days of fifth through eighth, October 2018, eleventh through sixteenth, October 2018. The cow 20112 was selected because she moved evenly over the pasture compared to the others and her data were unlikely biased as learning data. To do so, we first divided the pasture into $5 \mathrm{~m} \times 5 \mathrm{~m}$ grids and counted how many times each cow stayed in each grid area based on her location obtained by GPS device. And then, we calculated the coefficient of variation $C V$ of the times over all of the grids, which is given by

$$
C V=\frac{\sigma}{\bar{x}},
$$

where $\bar{x}$ denotes the average number of the times per grid and $\sigma$ does its standard deviation. If the value of $C V$ is small, it means that the cow moved evenly in the pasture. Finally, $C V$ was determined for all cows, i.e. 24 cows, and as a result, we have chosen the cow 20112 with the smallest $C V$ as learning data. 


\subsection{Learning method}

In this research, localization accuracy was compared in the following three learning methods.

1. Virtual data learning method: Learning method using only virtual data

2. Real data learning method: Learning method using only real data

3. Virtual and real data learning method: Learning method using real data after using virtual data

\section{Experiment and evaluation}

The test data used real data of the cow 20112, 20192, 20215, and 20295 in five days of 17 th through 21 st October 2018. Table I shows the result of localization of the matching approach and the deep learning approach using virtual data, and average processing time per one localization, average distance error, and standard deviation are summarized. In the matching approach, we used the most common classification algorithm given by

$$
\underset{k}{\arg \min }\left(\sum_{i=1}^{m} \sum_{j=1}^{n}\left(R S S I_{j}^{i}-R S S I_{j, k}^{i}\right)^{2}\right),
$$

where $k$ is the record number in the database as shown in Fig. 1, $m$ is the number of tags, $n$ is the number of antennas, $\operatorname{RSSI}_{j}^{i}$ is the measured RSSI, and $\operatorname{RSSI}_{j, k}^{i}$ is RSSI stored in the database. The estimated position $\left(x_{k}, y_{k}\right)$ is defined as the position stored in the record number $k$ satisfies Eq. (2). We used the error distance, $E$, between the GPS position $(x, y)$ and the estimated position $\left(x_{k}, y_{k}\right)$ to evaluate the error. The error distance, $E$, can be given by

$$
E=\sqrt{\left(x-x_{k}\right)^{2}+\left(y-y_{k}\right)^{2}} .
$$

Moreover, in Table I, 50 randomly selected from test data of the cow 20112 were used to evaluate the accuracy of localization. From this result, it can be seen that the deep learning approach can estimate the position faster and higher accuracy than the matching approach.

Table II shows the average distance error and its standard deviation when estimating the four cows' locations for test data. We can see that the virtual data learning method has larger average error and the standard deviation than the others. This is because of the fact that the virtual space cannot reflect an accurate real environment exactly, such as variations in the directivity of each antenna or irregularities in the grazing ground surface that are not completely horizontal. Moreover, the virtual and real data learning method can keep the average error and

Table I. Comparison result of the matching approach and the deep learning approach.

\begin{tabular}{lccc}
\hline $\begin{array}{l}\text { localization } \\
\text { approach }\end{array}$ & $\begin{array}{l}\text { Average localization } \\
\text { processing time }(\mathrm{s})\end{array}$ & $\begin{array}{l}\text { Average } \\
\text { error }(\mathrm{m})\end{array}$ & $\begin{array}{l}\text { Standard } \\
\text { deviation }(\mathrm{m})\end{array}$ \\
\hline \hline Matching & $7.75 \times 10^{2}$ & 30.84 & 16.27 \\
Deep learning & $7.36 \times 10^{-3}$ & 14.33 & 9.11 \\
\hline
\end{tabular}


Table II. Localization result in each learning method.

\begin{tabular}{llrrrr}
\hline \multirow{2}{*}{ Error evaluation (m) } & Learning method & \multicolumn{4}{c}{ Cow ID } \\
& & 20112 & 20215 & 20295 & 20192 \\
\hline \hline \multirow{2}{*}{ Average error } & Virtual data & 14.35 & 14.58 & 15.35 & 15.78 \\
& Real data & 6.08 & 6.63 & 7.32 & 7.18 \\
& Virtual \& real data & 5.87 & 6.16 & 6.48 & 7.33 \\
\hline \multirow{2}{*}{ Standard deviation } & Virtual data & 8.35 & 8.23 & 8.40 & 9.71 \\
& Real data & 3.61 & 3.92 & 5.85 & 4.23 \\
& Virtual \& real data & 3.59 & 3.78 & 4.12 & 4.72 \\
\hline
\end{tabular}

the standard deviation smaller than the real data learning method except for the cow 20192, and we realized outdoor localization with an average distance error of about $6 \mathrm{~m}$. From these results, it can be said that the effect of learning using the virtual space database from the viewpoint of generalization performance. Regarding the cause of large localization error of the cow 20192, we will further investigate the reason in the future.

\section{Conclusion}

In this paper, we proposed the localization method based on deep learning using virtual space. It is clarified that the deep learning approach is capable of localization with high speed and high accuracy compared with the matching approach. In addition, the virtual and real data learning method can keep the average error and the standard deviation smaller than the real data learning method, and we realized outdoor localization with an average distance error of about $6 \mathrm{~m}$.

\section{Acknowledgments}

This research was supported by JST CREST JPMJCR1682. 